\title{
Estimating the Burden of Irritable Bowel Syndrome: Analysis of a Nationwide Korean Database
}

\author{
Hye-Kyung Jung, ${ }^{1}$ Youn Hee Kim, ${ }^{2}$ Joo Yeon Park, ${ }^{2}$ Bo Hyoung Jang, ${ }^{2,3}$ Sun-Young Park, ${ }^{2,4}$ Mi-Hee Nam ${ }^{2}$ and Myung-Gyu Choi ${ }^{2,5 *}$ \\ ${ }^{1}$ Department of Internal Medicine, Ewha Womans University School of Medicine, Seoul, Korea; ${ }^{2}$ National Evidence-based Healthcare Collaborating \\ Agency, Seoul, Korea; ${ }^{3}$ College of Korean Medicine, Kyung Hee University, Seoul, Korea; ${ }^{4}$ School of Pharmacy, Sungkyunkwan University, \\ Suwon, Gyeonggi-do, Korea; and ${ }^{5}$ Department of Internal Medicine, College of Medicine, The Catholic University of Korea, Seoul, Korea
}

\section{Background/Aims}

Management of irritable bowel syndrome (IBS) imposes a heavy economic burden. This study was to estimate the epidemiologic features of IBS and to report the IBS burden for the first time in the Korean population.

\section{Methods}

A cross-sectional study was conducted using the National Health Insurance (NHI) system database, which covers the entire population of Korea. IBS was defined as diagnostic code -10 in adults with any outpatient clinic visits or hospitalization related to IBS. We excluded diseases that mimic IBS symptoms.

\section{Results}

A total of 2.42 million (58.2\% female) individuals were identified as patients with IBS, yielding an age- and gender-adjusted prevalence of $5.1 \%$ in males and $6.9 \%$ in females. The prevalence of IBS increased proportionally with age, with higher medical costs in middle-aged patients. Outpatient clinics were visited by $98.6 \%$ of IBS patients, and $1.9 \%$ were treated upon admission. Of these patients, $87.6 \%$ were given a prescription. Co-morbidities that commonly accompanied IBS included upper gastrointestinal $(36.1 \%)$, respiratory $(12.3 \%)$, musculoskeletal $(8.0 \%)$ disease, somatoform $(4.3 \%)$ and depression/anxiety disorders (3.1\%). The NHI costs of IBS, which include the NHI covered cost and beneficiary copayment charges, were estimated to be 155 million USD, which accounts for $0.46 \%$ of the total NHI costs for the entire Korean population.

\section{Conclusions}

According to the Korean national claims database, about $6 \%$ of the Korean population seeks medical care for IBS at least once per year. This high prevalence places a large economic burden on the Korean healthcare system, accounting for $0.46 \%$ of overall national medical expenditure.

\section{(J Neurogastroenterol Motil 2014;20:242-252)}

Received: October 25, 2013 Revised: January 23, 2014 Accepted: January 23, 2014

(c) This is an Open Access article distributed under the terms of the Creative Commons Attribution Non-Commercial License (http://creativecommons. org/licenses/by-nc/3.0) which permits unrestricted non-commercial use, distribution, and reproduction in any medium, provided the original work is properly cited.

*Correspondence: Myung-Gyu Choi, MD, PhD

Department of Internal Medicine, The Catholic University of Korea, College of Medicine, 222 Banpodae-ro, Seocho-gu, Seoul 137-701, Korea

Tel: +82-2-2258-6023, Fax: +82-2-2258-2055, E-mail: choim@catholic.ac.kr

Financial support: This manuscript was supported by the National Evidence-based Healthcare Collaborating Agency in $2009-2010$ (Grant No. NA09-018 and NA10-007).

Conflicts of interest: None.

Author contributions: Hye-Kyung Jung and Myung-Gyu Choi: study concept and design; Bo Hyoung Jang, Youn Hee Kim, Sun-Young Park and Mi-Hee Nam: acquisition of data; Bo Hyoung Jang and Joo Yeon Park: analysis and interpretation of data; Hye-Kyung Jung: drafting of the manuscript; Hye-Kyung Jung and Myung-Gyu Choi: critical revision of the manuscript for important intellectual content; Bo Hyoung Jang and Youn Hee Kim: statistical analysis; Myung-Gyu Choi and Hye-Kyung Jung: obtained funding; Hye-Kyung Jung: administrative, technical or material support; Myung-Gyu Choi: study supervision.

ORCID: Hye-Kyung Jung, http://orcid.org/0000-0002-6653-5214; Myung-Gyu Choi, http://orcid.org/0000-0003-4083-5187. 


\section{Introduction}

Irritable bowel syndrome (IBS) is a chronic condition characterized by recurrent abdominal pain or bowel symptoms but no structural or biochemical abnormalities. IBS can hinder daily activities, impair quality of life and impose a social and economic burden. It is remarkably common, affecting 3-20\% of the US population $^{1,2}$ and $2.3-9.6 \%$ of the Korean population. ${ }^{3-5}$ Despite its impact on public health, IBS has received relatively little attention compared to other life-threatening diseases. ${ }^{6}$

Healthcare decision makers, who establish payment policies and coverage, are increasingly seeking information about real-world outcomes as a basis for their decisions. The cost-effectiveness of interventions and efficient resource allocation should also be evaluated when making healthcare policy decisions. Previous studies of the economic burden of gastrointestinal (GI) disorders included only selected populations, ${ }^{7,8}$ because total-population or representative-sample data were not readily available. Previous studies also focused exclusively on the financial implications of diagnostic measures or therapeutic interventions, 9,10 rather than analyses of the entire specialty. ${ }^{10}$

In Korea, all citizens are automatically registered with the compulsory insurance program, the National Health Insurance (NHI) system which covers almost $100 \%$ of entire Korean population. The NHI allows citizens to access primary and secondary care without making an appointment in advance, as is necessary in Western countries. NHI also provides easy access to tertiary medical care at a lower cost. The Health Insurance Review \& Assessment (HIRA) is a service claims database of NHI which includes a nationwide, population-based health insurance electronic record of all medical and prescription drug claims in Korea. This database was used to conduct the present study, the aim of which was to evaluate the epidemiologic features of IBS in 2008 and the medical and socioeconomic burdens it imposes on the Korean population.

\section{Materials and Methods}

This cross-sectional study was conducted using HIRA, which covers the entire population of Korea, by extracting IBS-related data, excluding personal information. The study protocol was approved by the Institutional Review Board of the National Evidence-based Healthcare Collaborating Agency.

\section{Data Source}

The HIRA contains a computerized medical record system that includes general practices, secondary and tertiary hospitals, dental care and traditional Korean oriental medicine clinics. ${ }^{11}$ Under the NHI system, the HIRA electronic claims database covers $99.3 \%$ of outpatient health clinic usage and $93.9 \%$ of inpatient hospital treatment. ${ }^{11}$ The database broadly represents the Korean population in terms of sex, age and geography.

The information in the database includes demographics, clinical diagnoses, hospital discharge diagnoses and medical treatments provided-including prescriptions, investigational drugs, procedures and operations. ${ }^{11,12}$ Information regarding prescriptions includes brand names, quantities, durations and the Anatomical Therapeutic Chemical classification doses. ${ }^{13,14}$

\section{Identification of Individuals With Irritable Bowel Syndrome}

IBS is a common, often chronic, relapsing and remitting condition. In this study, we included all patients with IBS in the database from January 1 to December 31, 2008, beginning with the first day of medical care for each patient. Patients with IBS were identified as those who had at least one medical claim with a major primary code or the first four minor diagnostic codes based on the physician's diagnosis and discharge diagnostic codes. The reason for office visit is the principal reason given by the patient, which in the physician's judgment is the most appropriate one. However, identifying IBS cases solely based on diagnostic code can cause misclassification problems due to potential miscoding. To address this, we referred to the IBS-related information of each patient, including hospital visits, tests, and prescriptions. 
Overall, we defined IBS patients as those who had fulfilled all of the above criteria and who had incurred any medical expenses.

We included all outpatient claims or hospital admissions containing a diagnosis of IBS based on the International Statistical Classification of Diseases and Related Health Problems 10th Revision (ICD-10) ${ }^{15}$ codes K58 (IBS), K580 (IBS with diarrhea), or K589 (IBS without diarrhea). ICD-10 classifies disease, symptoms, abnormal findings, social circumstances and external causes of injuries with over 14,400 diagnostic codes, as classified by the World Health Organization. IBS is classified by subtype as diarrhea-predominant type (IBS-D), defined as K580 in the ICD-10 code, and non-diarrhea-predominant type (non-IBS-D), defined as any of following ICD-10 codes: K58, K589, K580 + $\mathrm{K} 589+\mathrm{K} 58, \mathrm{~K} 58+\mathrm{K} 589$ and K58 + K580.

\section{Exclusion Criteria}

In clinical practice, IBS is broadly defined as a multi-symptom complex characterized by abdominal pain or discomfort that is associated with altered bowel function. Therefore, we excluded individuals based on the following criteria (Figure): those younger than 15 years $(\mathrm{n}=676,512)$; individuals who had no medical expenses ( $\mathrm{n}=33,031)$; those with diagnostic codes of conditions that mimic IBS symptoms. These conditions included malignant neoplasm of the digestive organs $(n=34,047)$; inflammatory bowel diseases $(n=10,186)$; other acute enteritis/enterocolitis ( $n$ $=409,684)$; peptic ulcer disease $(\mathrm{n}=679,855)$; pancreato-biliary or liver cirrhosis ( $\mathrm{n}=37,106)$; genitourinary disease, such as urinary tract stones, cystitis, endometriosis or pelvic inflammatory disease $(n=94,303)$; intestinal obstruction or peritoneal diseases ( $\mathrm{n}=14,887)$; appendicitis $(\mathrm{n}=7,403)$; metabolic disease, including diabetes or thyroid disease $(n=203,991)$; and cerebrovascular disease or dementia $(n=30,992)$ (Table 1). Patients with extremely high medical costs were also reviewed and excluded when the cost of co-morbidities was prohibitively high (n $=10,849$ ). This was calculated as the mean high medical cost divided by the mean plus three standard deviations of each outpatient visit and hospital admission.

\section{Identification of Co-morbidities of Irritable Bowel Syndrome}

We identified co-morbidities using ICD-10 codes, more specifically, the major primary code or the first four minor diagnostic codes, based on the physician's diagnosis and discharge diagnostic codes. The co-morbidity codes were not mutually exclusive. To determine their prevalence, these conditions were categorized into intestinal and extra-intestinal co-morbidities. The former included gastroesophageal reflux disease or chest pain, other upper GI diseases (gastritis, dyspepsia, nausea and vomiting), or other intestinal diseases. Extra-intestinal diseases included musculoskeletal diseases (myalgia, joint pain, back pain or herniated nucleus pulposus), somatization (somatization disorder, symptoms of headache, sleep disturbance, tinnitus, fatigue, palpitation and

2008 The Korean health insurance review $\&$ assessment service (ICD-10 k58, k580, k589 as 1 st primary and 1st -4th secondary diagnostic codes) $(\mathrm{N}=4,659,270)$

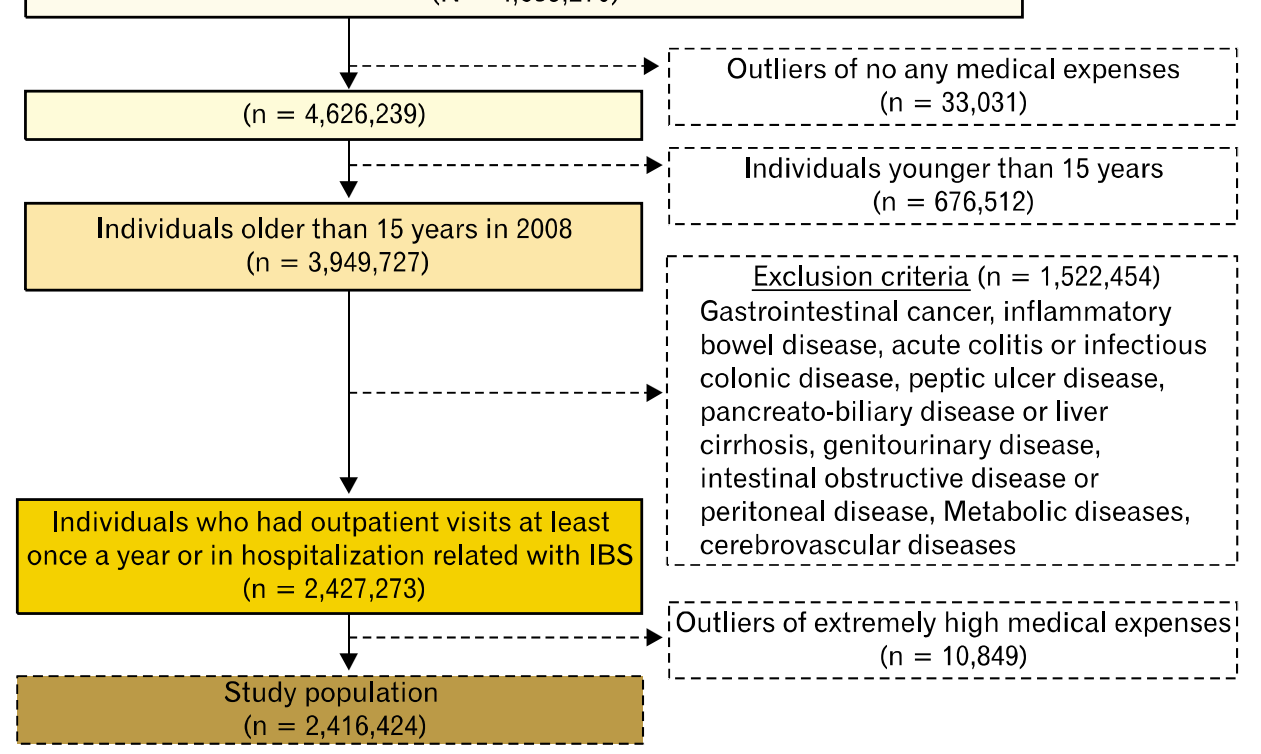

Figure. Study population: We included all outpatient claims or hospital admissions from the claim database of the National Health Insurance system. We excluded the patients having outliers of extremely no or high medical expenses, younger than 15 years or having diseases that mimic irritable bowel syndrome symptoms. 
Table 1. Diagnostic Codes of Excluded Organic Diseases

\begin{tabular}{|c|c|c|c|c|}
\hline Category of excluded disease & Diagnostic codes & $\operatorname{Men}(\mathrm{n}[\%])$ & Women (n [\%]) & Total (n [\%]) \\
\hline Gastrointestinal cancer & $\begin{array}{l}\text { Colon }(\mathrm{C} 18-\mathrm{C} 21) \\
\text { Esophagus or stomach }(\mathrm{C} 15, \mathrm{C} 16) \\
\text { Small bowel (C17) } \\
\text { Pancreato-biliary (C22-C25, C64) }\end{array}$ & $20,671(3.7)$ & $13,376(1.8)$ & $34,047(2.6)$ \\
\hline Inflammatory bowel disease & $\begin{array}{l}\text { Ulcerative colitis (K51) } \\
\text { Crohn's disease (K50) } \\
\text { Intestinal tuberculosis (A183, K930) }\end{array}$ & $5,302(0.9)$ & $4,884(0.7)$ & $10,186(0.8)$ \\
\hline $\begin{array}{l}\text { Acute colitis or } \\
\text { infectious colonic disease }\end{array}$ & $\begin{array}{l}\text { Gastritis/Enterocolitis (A09) } \\
\text { Other non-infectious gastritis/enterocolitis (K52) } \\
\text { Other infectious enterocolitis (A04) } \\
\text { Viral or other enteric infection (A08) } \\
\text { Other infectious food poisoning (A05) } \\
\text { Diverticulitis (K57) }\end{array}$ & $185,174(33.1)$ & $224,510(29.9)$ & $409,684(31.3)$ \\
\hline Peptic ulcer disease & $\begin{array}{l}\text { Gastric ulcer (K25) } \\
\text { Duodenal ulcer (K26) } \\
\text { Other unspecified peptic ulcer (K27-K28) }\end{array}$ & $294,280(52.6)$ & $385,575(51.4)$ & $679,855(51.9)$ \\
\hline $\begin{array}{l}\text { Pancreato-biliary disease or } \\
\text { liver cirrhosis }\end{array}$ & $\begin{array}{l}\text { Pancreato-biliary disease (K80-K87) } \\
\text { Hepatic fibrosis and cirrhosis (K74) }\end{array}$ & $20,001(3.6)$ & $17,105(2.3)$ & $37,106(2.8)$ \\
\hline $\begin{array}{l}\text { Genitourinary disease that mimics } \\
\text { intestinal bowel syndrome pain }\end{array}$ & $\begin{array}{l}\text { Urinary tract stone (N20-N23) } \\
\text { Cystitis (N30) } \\
\text { Pelvic inflammatory disease (N738-N739) } \\
\text { Endometriosis (N80) }\end{array}$ & $14,293(2.6)$ & $80,010(10.7)$ & $94,303(7.2)$ \\
\hline $\begin{array}{l}\text { Intestinal obstructive disease or } \\
\text { peritoneal disease }\end{array}$ & $\begin{array}{l}\text { Intestinal vascular disease (K55) } \\
\text { Paralytic ileus or mechanical intestinal obstruction (K56) } \\
\text { Postoperative intestinal obstruction (K913) } \\
\text { Peritoneal disease (K65-K67) }\end{array}$ & $6,474(1.2)$ & $8,413(1.1)$ & $14,887(1.1)$ \\
\hline Appendicitis & $\begin{array}{l}\text { Acute appendicitis, other appendicitis, unspecified } \\
\text { acute appendicitis (K35-K37) }\end{array}$ & $3,241(0.6)$ & $4,162(0.6)$ & $7,403(0.6)$ \\
\hline $\begin{array}{l}\text { Metabolic diseases influencing } \\
\text { gastrointestinal symptoms }\end{array}$ & $\begin{array}{l}\text { Hypothyroidism (K05) } \\
\text { Other hypothyroidism (E03) } \\
\text { Insulin-dependent diabetes mellitus (E10) } \\
\text { Non-insulin dependent diabetes mellitus (E11) } \\
\text { Unspecified diabetes mellitus (E13-E14) } \\
\text { Elevated blood glucose level (R73) }\end{array}$ & $87,990(15.7)$ & $116,001(15.5)$ & $203,991(15.6)$ \\
\hline $\begin{array}{l}\text { Cerebrovascular disease/mental } \\
\text { disease influencing } \\
\text { gastrointestinal symptoms }\end{array}$ & $\begin{array}{l}\text { Other mental disorders due to brain damage and } \\
\text { dysfunction and to physical disease (F06) } \\
\text { Cerebrovascular diseases (I60-I69) }\end{array}$ & $12,542(2.2)$ & $18,450(2.5)$ & $30,992(2.4)$ \\
\hline
\end{tabular}

dizziness), anxiety and depression, respiratory disease and other diseases.

\section{Cost Estimation}

The total cost of IBS was defined as the sum of direct and indirect costs (Supplementary Table 1). Direct costs are comprised of healthcare and pharmacy costs and transportation expenses. Healthcare costs were defined as the total expended cost in the health care institution, including the NHI covered and uncovered costs and beneficiary copayment. Covered NHI costs were retrieved directly from the database and compared with medical costs related to other diseases. These expenses included consultation fees, charges for diagnostic procedures (e.g., laboratory tests and radiological examinations), therapeutic procedures, and fees for inpatient and outpatient care. Uncovered NHI costs included sonography, meals, the endoscopy conscious sedation fee, extra fees for an upgraded hospital room, magnetic resonance imaging, positron emission tomography, and so on. Non-medical costs included only those related to transportation because traditional measures used in the context of cancer or heart failure, such as quality-adjusted life-years and days in bed, are less relevant in the context of IBS. Transportation fees were 
calculated as hospital visit days multiplied by the transportation fee based on the Korean National Statistical Office database. ${ }^{16}$

Indirect costs were defined as productivity costs in which sporadic work loss was associated with medical service use and extended work loss due to a disability or death. The time lost from work due to medical care was estimated by the type and frequency of visits to a health care institution. Each day in the hospital was considered a loss of a full work day and each outpatient visit was considered a loss of half a work day. In patients with IBS, IBS-related death was very rare. ${ }^{17}$ Therefore, productivity loss related to early death was not accounted for in this study. ${ }^{18}$ Presenteeism is a loss of productivity due to disease although the employee is present at work and absenteeism is a loss of work because the employee is absent from work due to disease. ${ }^{19}$ Indirect cost related with presenteeism or absenteeism was not included in this analysis because of limited data.

Sensitivity analysis was conducted to check the impact of the uncertain parameters in indirect costs (Supplementary Table 2). First, we assumed that employment population ratio of IBS patients was same as that of general populations. This is an optimistic assumption and we also performed sensitivity analysis assuming that employment population ratio of IBS patients was $20 \%$ lower than that of general populations. Second, we regarded that the lost time due to outpatient visit in IBS patients was half a work day, a usually applied method. As an alternative method, we used 3 hours instead of half a work day because IBS is not a fatal disease.

The original monetary values in Korean Won were converted into United States (US) dollars based on the average exchange rate in 2008 of 1 US dollar [USD] $=1,047.3$ Korean Won. Health care institutions were classified into primary clinics, secondary care hospitals, and general and teaching hospitals.

\section{Identification of Irritable Bowel Syndrome- related Diagnostic Test and Prescription Drugs}

IBS was defined by exclusion diagnosis. Therefore, we included all possible tests for exclusion of other GI or non-GI organic diseases. Also we included radiologic or endoscopic studies for upper GI tract because of overlap syndromes or co-morbidities. This included complete blood test and chemistry including serum amylase, serum lipase, carcinoembryonic antigen, urine analysis, thyroid function test, stool occult blood/ova test, simple abdominal X-ray, lower GI endoscopy, barium enema, upper GI endoscopy, upper GI studies, abdominal and/or pelvic CT scan and others. Since the costs of abdominal ultrasonography and magnetic resonance imaging are not covered by insurance, we did not include information regarding these procedures from the HIRA claim database.

Medications used for treatment of GI diseases included all drugs in the ATC classification system. ${ }^{13,14}$ These included anti-spasmodics, prokinetics, anti-diarrheals, laxatives, probiotics, anti-secretory agents, antacids, antibiotics, painkillers, and anxiolytics/anti-depressants.

\section{Statistical Methods}

We calculated the age- and sex-specific prevalence of IBS adjusted to the 2008 population demographics of South Korea, as obtained from the Korean National Statistical Office. ${ }^{16}$ Descriptive statistics were calculated based on variables such as medical utilization and cost measures, including numbers of inpatients and outpatients, physician office-visits and admissions, and laboratory and pharmacy claims.

\section{Results}

\section{Prevalence of Irritable Bowel Syndrome}

Among the 2008 Korean population who were at least 15 years of age, ${ }^{16}$ a total of 2,416,424 patients used medical care related to IBS during the 1 -year observation period. The prevalence of IBS in this population was $6.9 \%$ in females and $5.1 \%$ in males, yielding a female/male ratio of 1.44 (1,399,014 females, $57.9 \%$ ) (Table 2). IBS patients who visited outpatient clinics at least three times per year or were hospitalized totaled 487,179 (283,548 female, $58.2 \%$ ), a prevalence of $1.0 \%$ in males and $1.3 \%$ in females. There were no significant differences in IBS prevalence among the 10 administrative rural and urban districts in Korea. The proportions of medical specialties diagnosing IBS were $56.8 \%$ internal medicine, $21.8 \%$ general practice, $7.1 \%$ general surgery, $4.6 \%$ family medicine and $0.9 \%$ gynecology. We could not define the proportion by gastroenterologist because there was no separate data on gastroenterologist in NHI system. The prevalence of IBS tended to increase with age, ranging from $4.6 \%$ in $20-29$ year-olds to $8.7 \%$ in $60-69$ year-olds. However, the prevalence of IBS was lower in patients in their eighties than in those in their seventies.

We also categorized IBS according to subtype. The diarrhea predominant IBS (IBS-D) subtype was diagnosed in 862,585 patients $(33.8 \%)$. The proportion of IBS-D decreased with age with a prevalence of $48.1 \%$ in patients 20 years old or younger and 
Table 2. Age-Sex Specific Prevalence of Irritable Bowel Syndrome in 2008

\begin{tabular}{|c|c|c|c|c|c|c|c|c|}
\hline \multirow{3}{*}{ Age group (yr) } & \multicolumn{6}{|c|}{ Any medical service users with IBS $^{a}$} & \multicolumn{2}{|c|}{ Frequent medical service users with IBS } \\
\hline & \multicolumn{3}{|c|}{ No. of patients } & \multicolumn{3}{|c|}{ Prevalence (\%) } & \multirow{2}{*}{$\begin{array}{c}\text { No. of patients } \\
\text { All }\end{array}$} & \multirow{2}{*}{$\frac{\text { Prevalence }(\%)}{\text { All }}$} \\
\hline & All & Male & Female & All & Male & Female & & \\
\hline $15-19$ & 167,126 & 76,856 & 90,270 & 5.1 & 4.4 & 5.9 & 17,923 & 0.6 \\
\hline $20-29$ & 326,934 & 121,551 & 205,383 & 4.6 & 3.3 & 5.9 & 34,824 & 0.5 \\
\hline $30-39$ & 401,372 & 175,346 & 226,026 & 4.8 & 4.1 & 5.6 & 59,658 & 0.7 \\
\hline $40-49$ & 490,386 & 214,380 & 276,006 & 5.9 & 5.0 & 6.7 & 92,116 & 1.1 \\
\hline $50-59$ & 426,485 & 186,620 & 239,865 & 7.1 & 6.2 & 8.0 & 96,488 & 1.6 \\
\hline $60-69$ & 332,397 & 142,665 & 189,732 & 8.7 & 7.9 & 9.4 & 93,277 & 2.4 \\
\hline $70 \geq$ & 271,724 & 99,992 & 171,732 & 8.5 & 8.3 & 8.6 & 92,893 & 2.9 \\
\hline Total & $2,416,424$ & $1,017,410$ & $1,399,014$ & 6.0 & 5.1 & 6.9 & 487,179 & 1.2 \\
\hline
\end{tabular}

${ }^{a}$ Irritable bowel syndrome (IBS), defined as those who had at least one medical claim in outpatient clinic or hospitalization with a major primary code or minor 1st-4th diagnostic codes by physician's diagnosis and discharge diagnostic codes; ${ }^{b}$ Frequent visitors with IBS, defined as fulfillment of the above criteria and at least 3 visits to an outpatient clinic per year.

Table 3. Healthcare Utilization Related to Irritable Bowel Syndrome

\begin{tabular}{|c|c|c|c|c|c|c|c|c|}
\hline & \multicolumn{6}{|c|}{ Any medical service users with IBS } & \multicolumn{2}{|c|}{ Frequent medical service users with IBS } \\
\hline & \multicolumn{3}{|c|}{ No. of patients } & \multicolumn{3}{|c|}{ Proportion $(\%)^{\mathrm{a}}$} & \multirow{2}{*}{$\begin{array}{c}\text { No. of patients } \\
\text { All }\end{array}$} & \multirow{2}{*}{$\begin{array}{c}\text { Proportion (\%) } \\
\text { All }\end{array}$} \\
\hline & All & Male & Female & All & Male & Female & & \\
\hline \multicolumn{9}{|l|}{ Medical usage sector ${ }^{\mathrm{b}}$} \\
\hline Outpatients & $2,382,759$ & $1,001,934$ & $1,380,825$ & 98.6 & 98.5 & 98.7 & 453,514 & 93.1 \\
\hline Inpatients & 46,653 & 21,493 & 25,160 & 1.9 & 2.1 & 1.8 & 46,653 & 9.6 \\
\hline \multicolumn{9}{|c|}{ Type of healthcare institute ${ }^{b}$} \\
\hline Clinics & $1,891,235$ & 774,719 & $1,116,516$ & 78.3 & 76.1 & 79.8 & 413,820 & 84.9 \\
\hline Hospitals & 216,315 & 100,278 & 116,037 & 9.0 & 9.9 & 8.3 & 34,582 & 7.1 \\
\hline General hospitals & 291,649 & 132,645 & 159,004 & 12.1 & 13.0 & 11.4 & 59,047 & 12.1 \\
\hline Teaching hospitals & 73,785 & 33,649 & 40,136 & 3.1 & 3.3 & 2.9 & 18,069 & 3.7 \\
\hline
\end{tabular}

${ }^{a}$ Proportion calculated within the group, ${ }^{b}$ Classifications of medical usage sector and type of healthcare institute are not mutually exclusive. IBS, irritable bowel syndrome.

$31.7 \%$ in patients older than 60 years. IBS-D was also more prevalent in males $(38.3 \%)$ than in females $(33.8 \%)$ and decreased as higher-level of health care institutions were utilized, with a prevalence of $39.5 \%$ in primary clinics, $28.9 \%$ in secondary hospitals and $14.6 \%$ in referral centers, defined as general hospitals plus teaching hospitals.

\section{Healthcare Utilization Related to Irritable Bowel Syndrome}

Among the total patients with IBS, most patients visited outpatient clinics (98.6\%) and $1.9 \%$ of whom were treated upon admission. When categorized according to health care institution, the proportion of total IBS patients in primary clinics was $78.3 \%$ and that in referral centers, defined as general hospitals plus teaching hospitals, was $15.2 \%$ (Table 3). However, the pro- portion of IBS claims per total claims in referral centers (1.02\%) was similar to that in primary clinics $(0.87 \%)$. The mean $( \pm$ standard deviation) visit time for outpatients was $2.5 \pm 4.0$ days per year and that for inpatient care was $14.7 \pm 25.0$ days. Frequent medical service users who used healthcare at least three times per year visited the same healthcare center $78.5 \%$ of the time, two healthcare centers $18.5 \%$ of the time, and three healthcare centers $2.9 \%$ of the time.

\section{Co-morbidities of Irritable Bowel Syndrome}

We analyzed the 100 ranked diseases accompanying IBS as a major primary code or the first four minor diagnostic codes. Multiple co-morbidities were not mutually exclusive. Among the 9,880,510 co-morbidities, the diseases that most frequently accompanied IBS were upper GI diseases (36.1\%), followed by dis- 
Table 4. Total Costs of Irritable Bowel Syndrome

\begin{tabular}{|c|c|c|c|c|c|c|c|c|c|c|}
\hline \multirow{3}{*}{ Cost category } & \multirow{3}{*}{ Subsection } & \multirow{3}{*}{ Details } & \multicolumn{6}{|c|}{ Any medical service users with IBS ${ }^{a}$} & \multirow{2}{*}{\multicolumn{2}{|c|}{$\begin{array}{c}\text { Frequent users }^{\mathrm{b}} \\
\text { Total }\end{array}$}} \\
\hline & & & \multicolumn{2}{|c|}{ Outpatients } & \multicolumn{2}{|c|}{ Hospitalization } & \multicolumn{2}{|l|}{ Total } & & \\
\hline & & & Costs & $\%$ & Costs & $\%$ & Costs & $\%$ & Costs & $\%$ \\
\hline \multirow[t]{4}{*}{ Direct costs (A) } & & & $348,670,489$ & 74.2 & $71,654,254$ & 80.3 & $420,324,742$ & 75.2 & $239,224,768$ & 76.1 \\
\hline & Medical costs & Healthcare services $^{\mathrm{a}}$ & $149,894,403$ & 31.9 & $70,586,501^{\mathrm{c}}$ & 79.1 & $220,480,903^{\mathrm{a}}$ & 39.4 & $126,921,710$ & 40.4 \\
\hline & & Pharmacy $^{\mathrm{b}}$ & $113,621,831$ & 24.2 & $-^{c}$ & - & $113,621,831$ & 20.3 & $69,231,651$ & 22.0 \\
\hline & Non-medical costs & Transportation & $85,154,256$ & 18.1 & $1,067,753$ & 1.2 & $86,222,008$ & 15.4 & $43,071,407$ & 13.7 \\
\hline \multicolumn{3}{|c|}{ Indirect costs (productivity loss) (B) } & $121,063,582$ & 25.8 & $17,540,565$ & 19.7 & $138,604,147$ & 24.8 & $75,118,030$ & 23.9 \\
\hline \multicolumn{3}{|c|}{ Total $(A+B)$} & $469,734,070$ & 100.0 & $89,194,819$ & 100.0 & $558,928,890$ & 100.0 & $314,342,799$ & 100.0 \\
\hline
\end{tabular}

${ }^{\mathrm{a}}$ Healthcare service costs are the sum of National Health Insurance (NHI)-covered cost, beneficiary copayments and NHI-uncovered payments; ${ }^{\mathrm{b}} \mathrm{Pharmacy}$ costs are the sum of pharmaceutical costs for drugs and preparation fees for medications; ${ }^{c}$ Pharmacy costs during hospitalization are summed into the costs for healthcare services. IBS, irritable bowel syndrome.

Costs are presented in US dollars ( $1 \mathrm{USD}=1,047.3$ Korean won, 2008).

eases of the respiratory system (12.3\%), musculoskeletal system diseases $(8.0 \%)$, somatoform disorders $(4.3 \%)$ including sleep disturbance, headache, tinnitus, fatigue and palpitation, and depression/anxiety disorders (3.1\%).

\section{Costs Related to Irritable Bowel Syndrome}

The total costs of IBS in 2008 were estimated to be 559 million USD (Table 4). Of this, direct medical costs, including healthcare service and pharmacy costs, was 334 million USD, which accounted for $59.7 \%$ of the total costs of IBS. The costs of frequent medical service users with IBS were 314 million USD, which accounted for over $56 \%$ of total costs.

We performed the sensitivity analysis in assuming the indirect cost. When accounting the indirect cost by assumptions of diminishing $20 \%$ employment population ratio in IBS, the result was not sensitive. The indirect cost was 111 million USD, therefore, the total cost might be 531 million USD (5.0\% reduction of total cost). According to the change of time lost from work due to medical care as 3 hours instead of 4 hours, the total cost was 528 million USD ( $5.4 \%$ reduction of total cost).

NHI costs for IBS during 2008 were estimated to be 155 million USD, which corresponded to approximately $0.46 \%$ of the total costs for the entire Korean population, which was 33.4 billion USD. The annual average NHI costs for IBS per person were 64.1 USD (standard deviation 237.2 USD, median 19.1 USD). The outpatient costs totaled 43.7 USD and inpatient costs totaled 1087.9 USD. The mean NHI costs per person of females (60.8 USD) were lower than those of males (68.6 USD). However, the total costs for IBS in females were higher than those of males due to the prevalence of IBS in women. Medical costs were higher in patients of 40-59 years old, followed by those of $\geq 60$ years and 30-39 years old. The mean NHI costs per IBS patient were highest in teaching hospitals (181.6 USD), and lowest in primary clinics (36.1 USD). However, the sum of NHI costs for IBS in primary clinics was highest $\left(68.2 \times 10^{6}\right.$ USD in primary clinics, $44.9 \times 10^{6} \mathrm{USD}$ in general hospitals $14.0 \times 10^{6}$ USD in teaching hospitals). The mean NHI costs for frequent medical service users were twice those of total IBS patients.

\section{Utilization of Diagnostic Procedure Related to Irritable Bowel Syndrome}

Among the 4,889,145 claims related to IBS, 848,431 (17.4\%) utilized diagnostic procedures. Diagnostic procedures were performed more frequently in males (18.7\%) than females (16.4\%). The proportion of claims including diagnostic procedures was $12.2 \%$ in primary clinics, $41.9 \%$ in secondary clinics, $43.0 \%$ in general hospitals and $47.9 \%$ in teaching hospitals. Blood cell counts, chemistry tests and urinalyses were the tests most frequently used for patients with IBS (Table 5). Lower GI endoscopy was performed in $0.8 \%$ of total IBS claims and barium enema in $1.9 \%$ of total IBS claims. Upper GI endoscopy was undertaken in $18.7 \%$ among all IBS claims.

\section{Drug Utilization Related to Irritable Bowel Syndrome}

During 2008, 4,708,932 cases claimed medical care expenses for IBS treatment. Of these, 604,331 were excluded because no medication was used $(n=180,213)$ or because of a single use of anti-secretory agents, antacids, mucosal protectors, analgesics or antibiotics ( $n=424,118)$. Finally, the pharmaceutical usage and medical costs of 4,284,814 claims (87.6\%) were analyzed.

The most popular drugs in patients with IBS were anti-spas- 
Table 5. Utilization of Diagnostic Procedures Related to Irritable Bowel Syndrome in 2008

\begin{tabular}{|c|c|c|c|c|c|c|c|c|c|}
\hline & $\begin{array}{l}\text { Blood test }{ }^{\mathrm{a}} \\
\text { /urinalysis }\end{array}$ & $\begin{array}{c}\text { Simple } \\
\text { abdominal } \\
\text { X-ray }\end{array}$ & $\begin{array}{l}\text { Stool occult } \\
\text { blood/ova }\end{array}$ & $\begin{array}{l}\text { Barium } \\
\text { enema }\end{array}$ & $\begin{array}{c}\text { Lower } \\
\text { GI } \\
\text { endoscopy }\end{array}$ & $\begin{array}{c}\text { Upper } \\
\text { GI } \\
\text { endoscopy }\end{array}$ & CEA & $\begin{array}{l}\text { Thyroid } \\
\text { function test }\end{array}$ & $\begin{array}{c}\text { Abdominal } \\
\text { CT scan }\end{array}$ \\
\hline No. of procedures & 339,357 & 99,935 & 13,843 & 15,758 & 6,557 & 159,076 & 5,090 & 23,987 & 7,747 \\
\hline Percent & 40.0 & 11.8 & 1.6 & 1.9 & 0.8 & 18.7 & 0.6 & 2.8 & 1.2 \\
\hline Cumulative (\%) & 40.0 & 51.8 & 53.4 & 55.3 & 56.1 & 74.8 & 75.4 & 78.2 & 79.4 \\
\hline \multicolumn{10}{|l|}{ Gender } \\
\hline Male & 149,807 & 41,488 & 6,481 & 7,348 & 3,380 & 69,697 & 2,525 & 7,193 & 3,423 \\
\hline Female & 189,550 & 58,447 & 7,362 & 8,410 & 3,177 & 89,379 & 2,565 & 16,794 & 4,324 \\
\hline \multicolumn{10}{|l|}{ Age group (yr) } \\
\hline $15-19$ & 12,015 & 8,676 & 541 & 227 & 229 & 3,191 & 26 & 904 & 196 \\
\hline $20-29$ & 36,476 & 16,092 & 1,244 & 699 & 591 & 20,370 & 142 & 2,830 & 559 \\
\hline $30-39$ & 51,635 & 16,470 & 1,829 & 1,648 & 902 & 37,583 & 456 & 4,517 & 1,121 \\
\hline $40-49$ & 68,699 & 18,208 & 2,807 & 4,010 & 1,302 & 36,311 & 1,004 & 5,732 & 1,754 \\
\hline $50-59$ & 65,474 & 15,071 & 2,516 & 3,899 & 1,334 & 31,058 & 1,195 & 4,735 & 1,649 \\
\hline $60-69$ & 56,212 & 12,080 & 2,429 & 3,159 & 1,149 & 20,324 & 1,190 & 2,997 & 1,273 \\
\hline $70 \geq$ & 48,846 & 13,338 & 2,477 & 2,116 & 1,050 & 10,239 & 1,077 & 2,272 & 1,195 \\
\hline \multicolumn{10}{|c|}{ Type of healthcare institute } \\
\hline Clinics & 186,845 & 41,217 & 4,753 & 11,361 & 3,754 & 93,976 & 779 & 10,351 & 304 \\
\hline Hospitals & 45,640 & 23,719 & 2,341 & 1,600 & 540 & 19,549 & 737 & 3,836 & 907 \\
\hline General hospitals & 79,944 & 26,278 & 4,604 & 2,263 & 1,570 & 33,719 & 1,726 & 7,235 & 4,352 \\
\hline Teaching hospitals & 25,255 & 4,375 & 2,109 & 509 & 692 & 11,647 & 1,837 & 2,508 & 2,283 \\
\hline
\end{tabular}

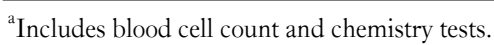

CEA, carcinoembryonic antigen.

Diagnostic procedures includes tests ordered in more than $0.01 \%$ of all claims. Percentage is the number of claims including each diagnostic procedure per the total number of orders of any diagnostic procedure $(\mathrm{n}=848,431)$.

modics, prescribed to $3,223,620(75.2 \%)$ of patients. Probiotics were used in $64.5 \%$ of patients, prokinetics in $43.5 \%$, anti-secretory agents in $35.6 \%$, analgesics in $20.0 \%$, anxiolytics in $8.7 \%$ and antidepressants in $6.8 \%$. The mean number of drugs prescribed for IBS was $6.0 \pm 5.6$, which increased with age but exhibited no gender difference. The number of drugs prescribed in referral hospitals was $8.7 \pm 12.8$ and in primary clinics was $5.5 \pm 2.8$.

Patients with IBS were frequently prescribed a combination of drugs due to the multi-symptom nature of IBS or a lack of effective therapies. Among 4,284,814 claims that included drug prescriptions, $34.1 \%$ were prescribed three categories of drugs (anti-spasmodics + anti-secretory agents + probiotics) and $27.3 \% 4$ categories of drugs (anti-spasmodics + prokinetics + anti-secretory agents + probiotics). Medical expenses related to prescription drug usage were 88,398,097 USD; this figure increased with age. Despite this, mean expenses per claim in primary clinics were lower than those in tertiary hospitals, and more than half of the total cost of drugs (57.9\%) was charged to primary clinics.

\section{Discussion}

We present here considerable data concerning the burden of IBS in Korea, including prevalence, hospital utilization and diagnostic and therapeutic costs, based on the HIRA claims database. A total of 2,416,424 patients used any medical care related to IBS at least once per year, which equated to 6 patients per 100 people aged 15 or older. Of these, $87.6 \%$ were prescribed medication, and most patients used outpatients' clinic and $2 \%$ received inpatient care, with a very low mortality rate. The direct economic burden of IBS was very high and comprised $0.46 \%$ of national medical care expenditures.

The prevalence of IBS was comparable to those reported in previous population-based studies (2.3-20\%). ${ }^{20-22}$ This variation in IBS prevalence may be due to the arbitrary definition that includes only the symptom complex and the varying thresholds associated with diagnostic symptom criteria. ${ }^{23}$ These limitations in the definition of IBS may confound assessment of the public health impact of the disease. In this respect, reporting the preva- 
lence of IBS using a national claim database might be a more consistent and practical assessment of disease burden.

After standardization of IBS by age distribution, the prevalence in females was 1.4 times that of males. However, this gender difference was less than that reported in Western countries. For example, IBS affects 1.5-3 times as many females as males in Europe, irrespective of the criteria used to define IBS. ${ }^{24}$ The IBS-D subtype was also more prevalent in males, which was consistent with other epidemiological studies in Asia. ${ }^{24-26}$ The highest medical costs in previous European studies were associated with older IBS patients, ${ }^{24}$ which might be related to more frequent hospital admission of older patients. However, in the present study, medical costs, resource utilization and claims were highest for middle-aged patients 40-59 years, which is consistent with data from the US. ${ }^{27}$ This may be due at least in part to economic incentives. Patients often visit physicians before receiving diagnosis of their IBS and undergo testing to exclude other conditions including colorectal cancer and inflammatory bowel disease. In contrast, older patients on fixed incomes tend to purchase medical services.

Data from the HIRA claims database in Korea confirm that IBS accounts for a substantial proportion of primary care physicians. Most IBS patients consult primary physicians including general practitioners, surgeons and gynecologists. This phenomenon is consistent in other countries; e.g., one European study reported that $28 \%$ of IBS patients consulted a gastroenterologist. $^{28}$ Over $80 \%$ of patients with IBS consulted a primary care physician. However, the proportion of the total claims represented by those related to IBS in referral centers was higher than that in primary clinics, suggesting that IBS is a common and important disorder not only in primary clinics but also in tertiary care centers in Korea. Half of patients with IBS in referral hospitals underwent diagnostic tests, compared to one in ten in primary clinics. Interestingly, lower GI study was conducted in $2.7 \%$ of total IBS claims, but upper GI endoscopy was in $18.7 \%$ of total IBS claims. It might be related with comorbidities or overlap syndrome of functional dyspepsia in one-third of IBS patients. This also explains why there are so many antisecretory drug prescriptions (35.6\%) in this study. In Korea, upper GI endoscopy is most popular diagnostic tool for upper GI symptoms because of high gastric cancer prevalence and accompanying endoscopic screening policy as a result. The routine check-up of upper endoscopy is offered free of charge to all Korean over age 40 at least every other year.

Our findings indicate that the high prevalence of IBS in
Korea imposes a substantial economic burden. Comparing direct expenditures among countries is difficult due to differences in healthcare management systems. The United Kingdom (UK) has adopted a National Health Service model. ${ }^{29}$ In 1995, the UK spent 45.6 million pounds $(£)$ in total IBS expenditure. This constituted $0.1 \%$ of the total annual spending by the UK National Health Service. The US healthcare system is based on consumer sovereignty or a private insurance model. The US spent $0.5 \%$ of total direct costs for functional GI disorders (FGIDs), including IBS, functional dyspepsia, constipation, and biliary colic. The healthcare system in Korea is a compromise between the UK and the US systems, with the twin aims of accessibility and good insurance coverage.

Medical charges in Korea are lower than that of the US and the UK. For example, an average colonoscopy costs \$95-150 in Korea, whereas it costs $\$ 3,081$ in the $\mathrm{US}^{30}$ and $£ 1005-2195$ in the UK. ${ }^{31}$ Despite the less expensive charges in Korea, the total direct costs based on national health expenditure are similar to those in the UK and the US, reflecting the more liberal use of the Korean healthcare system. This study demonstrated that Korean IBS patients visited outpatient clinics more frequently, at a rate of 2.5 per year compared to 1.6 in the UK and 0.9 in the US. ${ }^{32}$

Health care cost collection and estimation can be challenging. One of the most important components of cost collection is defining the cost perspective or reference point of any given analysis. The cost analysis includes accurate overall costs (direct cost) and accurate statistical tests to handle uncertainty (indirect cost). Because of the inherent imperfections in research data, statistical tests and sensitivity analysis to evaluate data uncertainty are a critical component to all economic evaluations. The indirect costs in patients with IBS accounted for two-thirds of the total in industrialized countries. ${ }^{30,31}$ In contrast, indirect costs were assumed to be $25 \%$ in the present study because the costs related to presenteeism and absenteeism due to IBS were not included. In Korea, there are limited data on measuring the indirect cost related with IBS, especially the productivity costs related presenteeism and absenteeism. However, sensitivity analysis revealed that the indirect cost only varied within the range of $5 \%$ of total cost according to the change of employment ratio or time lost from work. To estimate these costs, the further study, such as the patient survey including "Work Productivity and Activity Impairment" will be necessary. ${ }^{19}$

Similar to Western countries, in which $23.4 \%$ of IBS direct costs are attributed to medication, ${ }^{7}$ in Korea IBS-related direct medical costs comprised $19.0 \%$. In particular, patients with IBS 
were prescribed 6 kinds of GI-related drugs, including anti-spasmodics, prokinetics, probiotics, anxiolytics, proton pump inhibitors and antacids. A substantial number of patients with IBS also had upper GI disease (36.1\%), including FGIDs. It might be related with significant overlap of FGIDs. In addition, combination therapy was frequently used in IBS patients due to symptom heterogeneity. ${ }^{33,34}$

In contrast, a third of the total direct medical costs were consumed by in-patient stays for evaluation, diagnosis and treatment. ${ }^{35}$ Distribution of medical costs for IBS patients was skewed by high healthcare utilization. Frequent healthcare users who visited healthcare providers at least three times per year or who were hospitalized with IBS, comprised $20 \%$ of the total number of IBS patients but accounted for two-thirds of the total medical provider charges for IBS.

Medical utilization data for either the total population or a representative sample are not readily available. The majority of population-based studies on healthcare utilization have some limitations because most involve a relatively small number of individuals and are prone to recall bias since they rely mainly on self-reporting of IBS-related consultations by subjects. Reports of economic assessments in gastroenterology deal mainly with the cost-effectiveness of single pharmaceuticals, diagnostic measures or therapeutic interventions. The strength of the present study was that we used the complete claim data of an entire population and included primary, secondary and referral medical centers. All data are completely drawn from single dataset; demographic data, physician's diagnostic and therapeutic services ordered or provided, diagnosis and drug prescribed are included. The nation-wide single claim data can exclude the duplication data. Therefore, these data have strong generalizability and avoid selection bias. In addition, our data, which are based on the NHI claim database, compare the IBS-related disease burden with those of other significant diseases. The direct medical costs of IBS in healthcare institutes amounted to 334 million USD. This is about $30 \%$ of those related to cerebrovascular accidents (1,071 million USD), ${ }^{36}$ but higher than those of depression (125.8 USD) $)^{37}$ or asthma (116.4 USD). ${ }^{38}$ Therefore, IBS imposes a high economic burden.

Other limitations may have arisen from use of administrative claim data. First, prevalence based on insurance claims records could be underestimated because some IBS patients do not seek medical management. Our data did not include insurer-uncovered costs, private insurance, alternative medicine, and non-medical treatment such as psychological or behavioral therapy. However, insurer-uncovered costs are relatively small because most structural tests related to IBS are covered by
HIRA, except abdomen ultrasonography and magnetic resonance radiography. Second, IBS diagnosis was made by doctors using ICD-codes for billing reasons. Therefore, diagnosis was based on clinical assessment, which did not necessarily apply Rome criteria. The reliability of ICD-10 diagnostic codes for identification of IBS might be not high because of the inherent nature of claims data; i.e., voluntary or non-voluntary miscoding behaviors. Therefore, among 4,430,104 patients with an initial query of IBS, we excluded 1,309,735 subjects who may have had other organic diseases and included IBS patients with medical expenses. Furthermore, a recent study that aimed to validate the diagnostic codes of the HIRA claims database reported that $70 \%$ of such diagnostic codes coincide with those in medical records. ${ }^{11}$

A better understanding of the determinants of medical costs can lead to cost-saving strategies. Our study indicates that co-morbidities and the lack of effective therapy increase the estimated direct healthcare costs. Therefore, these are potential targets for healthcare expenditure reduction strategies. Effective therapy for IBS seemed like an elusive goal, probably by IBS presenting with too diverse target for clinical research. However, further well-conducted studies with realistic subgrouping, consideration of stratification for comorbidities and well-designed endpoint may provide more reliable evidence regarding heterogeneous symptoms of patients with IBS. The systemic and comprehensive support to researches of FGIDs should be another step to reach the optimal care of IBS and finally reduce the economic burden. Our findings also confirmed that a wide range of physicians, including general practitioners, gastroenterologists, general surgeons and gynecologists, are involved in the care of patients with manifestations of IBS. Approaches to reduce direct costs include education of physicians and patients and development of IBS management guidelines.

\section{Supplementary Materials}

Note: To access the supplementary tables mentioned in this article, visit the online version of Journal of Neurogastroenterology and Motility at http://www.jnmjournal.org/, and at doi: http://dx.doi.org/10.5056/jnm.2014.20.2.242.

\section{References}

1. Drossman DA, Li Z, Andruzzi E, et al. U.S. householder survey of functional gastrointestinal disorders. Prevalence, sociodemography, and health impact. Dig Dis Sci 1993;38:1569-1580.

2. Whitehead WE, Palsson O, Jones KR. Systematic review of the comorbidity of irritable bowel syndrome with other disorders: what are 
the causes and implications? Gastroenterology 2002;122:1140-1156.

3. Nam SY, Kim BC, Ryu KH, Park BJ. Prevalence and risk factors of irritable bowel syndrome in healthy screenee undergoing colonoscopy and laboratory tests. J Neurogastroenterol Motil 2010;16:47-51.

4. Jeong JJ, Choi MG, Cho YS, et al. Chronic gastrointestinal symptoms and quality of life in the Korean population. World J Gastroenterol 2008;14:6388-6394.

5. Park DW, Lee OY, Shim SG, et al. The differences in prevalence and sociodemographic characteristics of irritable bowel syndrome according to Rome II and Rome III. J Neurogastroenterol Motil 2010;16:186-193.

6. Talley NJ, Gabriel SE, Harmsen WS, Zinsmeister AR, Evans RW. Medical costs in community subjects with irritable bowel syndrome. Gastroenterology 1995;109:1736-1741.

7. Leong SA, Barghout V, Birnbaum HG, et al. The economic consequences of irritable bowel syndrome: a US employer perspective. Arch Intern Med 2003;163:929-935.

8. Levy RL, Von Korff M, Whitehead WE, et al. Costs of care for irritable bowel syndrome patients in a health maintenance organization. Am J Gastroenterol 2001;96:3122-3129.

9. Suleiman S, Sonnenberg A. Cost-effectiveness of endoscopy in irritable bowel syndrome. Arch Intern Med 2001;161:369-375.

10. Chou LF. Estimating medical costs of gastroenterological diseases. World J Gastroenterol 2004;10:273-278.

11. Kim JY. National health insurance data to vitalize the evidence-based health care: the current status and tasks. Health Technology Assessment Sourcebook in Health Insurance Review \& Assessment Service 2005.The 4th Statistical Innovation Forum:17-52.

12. Everhart JE, Ruhl CE. Burden of digestive diseases in the United States part I: overall and upper gastrointestinal diseases. Gastroenterology 2009;136:376-386.

13. WHO Collaborating Centre for Drug Statistics Methodology. ATC/DDD Index. Available from URL: http://www.whocc.no/atc_ ddd_index (accessed 5 March 2014).

14. WHO Collaborating Centre for Drug Statistics Methodology. Guidelines for ATC classification and DDD assignment. Available from URL: http://www.whocc.no/atc_ddd_publications/guidelines (accessed 5 March 2014).

15. World Health Organization. International statistical classification of diseases and related health problems 10th revision version for 2010 . Available from URL: http://apps.who.int/classifications/apps/icd/ icd10online (accessed 5 March 2014).

16. Korean Statistical Information Service. Available from URL: http:// kosis.kr/ (accessed 5 March 2014).

17. Chang JY, Locke GR 3rd, McNally MA, et al. Impact of functional gastrointestinal disorders on survival in the community. Am J Gastroenterol 2010;105:822-832.

18. Cash B, Sullivan S, Barghout V. Total costs of IBS: employer and managed care perspective. Am J Manag Care 2005;11(Suppl):S7-S16.

19. Reilly MC, Bracco A, Ricci JF, Santoro J, Stevens T.The validity and accuracy of the Work Productivity and Activity Impairment questionnaire - irritable bowel syndrome version (WPAI:IBS). Aliment Pharmacol Ther 2004;20:459-467.

20. Gwee KA, Bak YT, Ghoshal UC, et al. Asian consensus on irritable bowel syndrome. J Gastroenterol Hepatol 2010;25:1189-1205.

21. Saito YA, Schoenfeld P, Locke GR 3rd. The epidemiology of irrita- ble bowel syndrome in North America: a systematic review. Am J Gastroenterol 2002;97:1910-1915.

22. Gwee KA, Wee S, Wong ML, Png DJ. The prevalence, symptom characteristics, and impact of irritable bowel syndrome in an asian urban community. Am J Gastroenterol 2004;99:924-931.

23. Boyce PM, Koloski NA, Talley NJ. Irritable bowel syndrome according to varying diagnostic criteria: are the new Rome II criteria unnecessarily restrictive for research and practice? Am J Gastroenterol 2000;95:3176-3183.

24. Quigley EM, Bytzer P, Jones R, Mearin F. Irritable bowel syndrome: the burden and unmet needs in Europe. Dig Liver Dis 2006;38:717-723.

25. Tang YR, Yang WW, Wang YL, Lin L. Sex differences in the symptoms and psychological factors that influence quality of life in patients with irritable bowel syndrome. Eur J Gastroenterol Hepatol 2012;24:702-707.

26. Kellow JE. Pathophysiology and management of irritable bowel syndrome. Korean J Intern Med 2001;16:137-146.

27. Nyrop KA, Palsson OS, Levy RL, et al. Costs of health care for irritable bowel syndrome, chronic constipation, functional diarrhoea and functional abdominal pain. Aliment Pharmacol Ther 2007;26:237-248.

28. Hungin AP, Whorwell PJ, Tack J, Mearin F. The prevalence, patterns and impact of irritable bowel syndrome: an international survey of 40,000 subjects. Aliment Pharmacol Ther 2003;17:643-650.

29. Fullerton S. Functional digestive disorders (FDD) in the year 2000--economic impact. Eur J Surg 1998;164(suppl 12):62-64.

30. How much does a colonoscopy cost? Available from URL: http://health.costhelper.com/colonoscopy.html (accessed 5 March 2014).

31. Colonoscopic costs in the UK. Available from URL: http://www. privatehealth.co.uk/hospitaltreatment/whatdoesitcost/colonoscopy/ (accessed 5 March 2014).

32. Camilleri M, Choi MG. Review article: irritable bowel syndrome. Aliment Pharmacol Ther 1997;11:3-15.

33. Noh YW, Jung HK, Kim SE, Jung SA. Overlap of erosive and non-erosive reflux diseases with functional gastrointestinal disorders according to Rome III criteria. J Neurogastroenterol Motil 2010;16: 148-156.

34. Jung HK, Halder S, McNally M, et al. Overlap of gastro-oesophageal reflux disease and irritable bowel syndrome: prevalence and risk factors in the general population. Aliment Pharmacol Ther 2007;26: 453-461.

35. Sandler RS, Everhart JE, Donowitz M, et al. The burden of selected digestive diseases in the United States. Gastroenterology 2002;122: 1500-1511.

36. Lim SJ, Kim HJ, Nam CM, et al. Socioeconomic costs of stroke in Korea: estimated from the Korea National Health Insurance Claims Database. J Prev Med Public Health 2009;42:251-260.

37. Kim N, Kim K, Lee S, et al. Medical use and quality level of patients with depression. Health Insurance Review \& Assessment Sevice 2008;3. Available from URL: http://www.hira.or.kr/rci_icenter. inter.do?action $=$ listStudy Info\&fldcd $=$ A $1 \&$ pgmid $=$ HIRAA030095010000 (accessed 5 March 2014).

38. Park CS, Kang HY, Kwon I, Kang DR, Jung HY. Cost of illness study of asthma in Korea: estimated from the Korea National Health Insurance Claims Database. J Prev Med Public Health 2006;39:397-403. 